\title{
A judicialização da saúde no Distrito Federal, Brasil
}

\author{
The judicialization of health in the Federal District of Brazil
}

Debora Diniz ${ }^{1}$

Teresa Robichez de Carvalho Machado ${ }^{2}$

Janaina Penalva ${ }^{1}$

\footnotetext{
${ }^{1}$ Programa de PósGraduação em Política Social, Universidade de Brasília. Caixa Postal 8011. 70.673-970 Brasília DF Brasil.d.diniz@anis.org.br ${ }^{2}$ Faculdade de Direito, Universidade de Brasília.
}

\begin{abstract}
This paper seeks to analyze the Judiciary's approach with respect to demands for the judicialization of the right to health by means of a case study of civil lawsuits for access to health care in Brazil's Federal District. Judicialization of the right to health signifies the judicialization of various of the health services provided. This is a descriptive and exploratory case study that covers the Federal District and uses mixed techniques to gather and analyze data. This study analyzed 385 lawsuits (87\% of the total number of cases of judicialization of health for the period from 2005 to 2010 that reached the Appellate court). The results indicate that the most judicialized service is access to intensive care unit, followed by drugs and health care. Almost all lawsuits are filed by public defenders, with medical prescriptions and recommendations from the public health service. The results of this study challenge some dominant themes in the national debate, particularly the claim that judicialization is a phenomenon of the elites and that the services judicialized are drugs. The study does not seek to make generalizations, but highlights the fact that the phenomenon of judicialization of health has different aspects encompassed under the same concept.

Key words The judicialization of health, Judicial decisions, Right to health, Health policies
\end{abstract}

Resumo Este artigo discute as tendências do Poder Judiciário frente às demandas sobre judicialização do direito à saúde, por meio de um estudo de demandas judiciais cíveis por acesso à assistência em saúde no Distrito Federal. Por judicialização do direito à saúde, entende-se a judicialização de variadas prestações de saúde. Trata-se de um estudo exploratório, descritivo, longitudinal, com uso de técnicas mistas de levantamento e análise dos dados coletados no DF. Foram analisadas 385 ações, o que corresponde a $87 \%$ do total de casos de judicialização da saúde do período entre 2005 e 2010 que alcançaram a 2a Vara. Os resultados indicam que o principal bem judicializado é o acesso à UTI, seguido por medicamentos e assistência médica. A quase totalidade dos processos é apresentado por defensor público, com receitase indicações médicas oriundas do serviço público de saúde. Em cerca de 8\% dos casos, há comprovante de renda no processo com predominância dos valores em torno de $R \$ 500,00$. Os resultados da pesquisa desafiam algumas teses dominantes no debate nacional, em particular a alegação de que é um fenômeno das elites e que o bem judicializado são os medicamentos. Os dados não têm pretensões generalizantes, mas aponta que o fenômeno da judicialização da saúde tem diferentes aspectos englobados pelo mesmo conceito.

Palavras-chave Judicialização da Saúde, Decisões judiciais, Direito à saúde, Políticas de saúde 


\section{Introdução}

A judicialização da saúde é uma questão recente no Brasil ${ }^{1-5}$. Por judicialização da saúde, deve-se entender uma questão ampla e diversa de reclame de bens e direitos nas cortes: são insumos, instalações, medicamentos, assistência em saúde, entre outras demandas a serem protegidas pelo princípio do direito à saúde. A origem da questão é ainda incerta, não apenas pela ausência de estudos empíricos sistemáticos e comparativos no país, mas principalmente pela amplitude da judicialização e seus diferentes níveis de expressão nas cortes. Em consequência, existem argumentos distintos para analisar as implicações da judicialização do direito à saúde no país: de um lado, se anuncia a possibilidade de efetivação do direito, mas, por outro, há o risco de a judicialização ser uma interferência indevida do Judiciário nas políticas públicas caso a decisão judicial não adote critérios objetivos e uniformes ou não seja munida de informações suficientes para uma correta avaliação quanto à viabilidade e adequação técnica e orçamentária do bem demandado ${ }^{1,6,7}$.

Alguns estudos nacionais indicam que o principal bem judicializado nas cortes são os medicamentos. Estes dados devem ser considerados com cautela, uma vez que adotam diferentes metodologias de recuperação das evidências e não há estudos comparativos ou de âmbito nacional ${ }^{3,8-11}$. A política de assistência farmacêutica apresenta uma série de desafios quanto ao desenho, operacionalização, atualização das listas de medicamentos distribuídos pelo Sistema Único de Saúde, transparência de protocolos clínicos, ausência de instâncias recursais, celeridade nas decisões, articulação com outras esferas de fiscalização e registro ${ }^{12-14}$. No entanto, não é toda demanda por assistência em saúde que decorre de necessidades, por isso, nem toda demanda deve ser atendida pelas políticas de saúde. Entre necessidade, demanda e acesso há o desenho da política com os critérios de eficácia, eficiência, segurança e uso racional dos recursos e da informação ${ }^{15}$.

Esses desafios podem se caracterizar como falhas da política (por exemplo, se o bem demandado já é objeto de uma política pública, mas sua obtenção não foi possível ou foi inadequada através da esfera administrativa), ou mesmo entraves à compreensão da política para sua operacionalização nas diferentes esferas do Poder Público, e, como resultado, a judicialização tanto pode ser um recurso para a garantia do justo em saúde quanto uma interferência indevida do Poder Judiciário no funcionamento da política de saúde.
Além disso, no conjunto do que se conhece como judicialização da saúde, há também a judicialização da assistência em saúde, ou seja, há casos em que o indivíduo procura as cortes para garantir seu acesso ao Sistema Único de Saúde. Apesar de pouco estudado no Brasil, não se deve confundir esses dois bens judicializados, pois apontam para questões diferentes nas cortes ${ }^{16}$.

A vasta maioria dos países com sistema universal de saúde não garante o direito à saúde, mas o direito aos serviços de saúde ${ }^{17,18}$. O Brasil experimenta um quadro original no cenário internacional da última década de judicialização da política de saúde em países com sistemas universais públicos - aqui, o bem protegido é o direito à saúde. Há um caráter pouco evidente do conceito de saúde para a resolução deste conflito. Parte importante da judicialização do direito à saúde não visa garantir o direito à vida, mas sustenta-se em outro princípio ainda mais abstrato, que é o da dignidade da pessoa humana. A saúde não seria um fim em si mesmo, mas um meio para a garantia da dignidade ${ }^{19}$. É exatamente nesse contexto argumentativo e de encontro tenso entre a implementação da política e a busca crescente do Poder Judiciário para acesso à assistência em saúde que este artigo analisa o fenômeno da judicialização da saúde tendo o Distrito Federal como unidade de análise.

\section{Metodologia}

O levantamento de dados foi feito na $2^{\text {a }}$ Vara de Fazenda Pública Privativa do Tribunal de Justiça do Distrito Federal e Territórios, cuja atribuição é julgar os feitos em que o Distrito Federal figura como parte interessada. O DF conta com oito varas de Fazenda Pública e a distribuição dos processos ocorre de maneira aleatória entre as unidades. As razões para a escolha do DF como unidade de análise foram duas: a ausência de estudos empíricos sobre a judicialização da saúde e a escassez oficial de leitos de UTI em hospitais públicos de saúde. Além disso, o mecanismo de distribuição dos processos de forma aleatória entre as Varas de Fazenda Pública permitia que a recuperação dos processos em uma única Vara garantisse ao estudo o caráter de aleatoriedade no levantamento de dados. A análise dos dados cobriu o período de maio de 2005 a setembro 2010, data do primeiro processo sobre saúde que alcançou a Vara e o primeiro dia do trabalho de campo para a pesquisa.

O trabalho de campo foi realizado durante 10 semanas, de forma presencial na Vara por três 
pesquisadoras. Foi utilizado um instrumento eletrônico para a coleta de dados, composto de 26 questões fechadas e estruturadas e 11 questões abertas. $\mathrm{O}$ instrumento foi validado em uma amostra de $40 \%$ dos processos. Após a validação, foram recuperados e analisados 385 processos, o que corresponde a $87 \%$ do universo total de processos sobre saúde tramitados na Vara no período integral da pesquisa.

A recuperação dos processos foi realizada em duas etapas. Na primeira, levantou-se todos os processos cuja distribuição indicava referir-se à saúde. Essa indexação inicial é produzida pela área de estatística do TJDFT e fornecida aos pesquisadores. Foram recuperados 533 processos sob essa rubrica. A segunda etapa consistiu na avaliação do objeto da judicialização e resultou na eliminação de 90 processos por erro de indexação, por isso o universo potencial de pesquisa foi estabelecido em 443 processos. No total, foram analisados 385 processos, havendo uma perda de 58 por razões diversas, sendo as mais comuns a sua posse pelo advogado, tramitação em regime de urgência ou análise pelo Ministério Público. Como não há prazo para o retorno do processo à Vara, a pesquisa em profundidade foi realizada no universo de 385 unidades, o que corresponde a $87 \%$ do total sobre o tema da saúde julgados pela 2a Vara de Fazenda Pública Privativa do TJDFT no período de 2005 a 2010.

\section{Tabelas e resultados}

A Tabela 1 apresenta as variáveis constantes dos processos.

Há um crescimento permanente da judicialização nos últimos cinco anos. Verifica-se através dos dados o aumento do número de processos impetrados nos últimos anos, somente os três últimos anos pesquisados contam com 344 (89\%). A judicialização da saúde no Distrito Federal é provocada por homens (51\%) e mulheres (46\%), de recém-nascidos a idosos com 101 anos (aproximadamente metade da população tem 59 anos ou menos), cujo pedido inicial é encaminhado por defensores públicos (95\%), com receita médica oriunda de serviços públicos de saúde (85\%).

Não há evidências de que a judicialização seja um movimento das elites. Praticamente todos os processos $(95 \%)$ foram conduzidos pela defensoria pública, o que pressupõe a hipossuficiência de recursos. Apenas $4 \%$ tiveram condução por um escritório de advocacia privada. Outros tipos de advocacia, como advogados de ONG, es-
Tabela 1. Variáveis constantes dos processos.

\begin{tabular}{|c|c|c|}
\hline & $\mathbf{N}$ & $\%$ \\
\hline \multicolumn{3}{|l|}{ Fluxo de ações no tempo - ano } \\
\hline 2005 & 5 & 1 \\
\hline 2006 & 10 & 3 \\
\hline 2007 & 26 & 7 \\
\hline 2008 & 58 & 15 \\
\hline 2009 & 120 & 31 \\
\hline 2010 & 166 & 43 \\
\hline Total & 385 & 100 \\
\hline \multicolumn{3}{|l|}{ Sexo } \\
\hline Masculino & 197 & 51 \\
\hline Feminino & 178 & 46 \\
\hline Não informado & 10 & 3 \\
\hline Total & 385 & 100 \\
\hline \multicolumn{3}{|l|}{ Faixa etária (anos) } \\
\hline 0 a 9 & 50 & 13 \\
\hline 10 a 19 & 14 & 4 \\
\hline 20 a 29 & 28 & 7 \\
\hline 30 a 39 & 35 & 9 \\
\hline 40 a 49 & 51 & 14 \\
\hline 50 a 59 & 47 & 12 \\
\hline 60 a 69 & 43 & 11 \\
\hline 70 a 79 & 67 & 17 \\
\hline Acima de 80 & 23 & 6 \\
\hline Não identificado & 27 & 7 \\
\hline Total & 385 & 100 \\
\hline \multicolumn{3}{|l|}{ Origem da Prescrição } \\
\hline No serviço público & 326 & 85 \\
\hline No serviço privado & 33 & 9 \\
\hline Não é possível identificar & 21 & 5 \\
\hline Não há pedido médico & 5 & 1 \\
\hline Total & 385 & 100 \\
\hline \multicolumn{3}{|l|}{ Tipo de advocacia dos processos } \\
\hline Defensoria Pública & 368 & 95 \\
\hline Escritório particular & 14 & 4 \\
\hline $\begin{array}{l}\text { Escritório modelo, Advogado de } \\
\text { ONG e Ministério Público Federal }\end{array}$ & 3 & 1 \\
\hline Total & 385 & 100 \\
\hline \multicolumn{3}{|l|}{ Comprovação de Renda } \\
\hline Abaixo de $\mathrm{R} \$ 500,00$ & 10 & 37 \\
\hline Entre $\mathrm{R} \$ 500,00$ e $\mathrm{R} \$ 999,00$ & 9 & 33 \\
\hline Entre $\mathrm{R} \$ 1.000,00$ e $\mathrm{R} \$ 1.499,00$ & 5 & 18 \\
\hline Entre $\mathrm{R} \$ 2.000,00$ e $\mathrm{R} \$ 2.999,00$ & 2 & 8 \\
\hline Entre $\mathrm{R} \$ 3.000,00$ e $\mathrm{R} \$ 4.000,00$ & 1 & 4 \\
\hline Total & 27 & 100 \\
\hline
\end{tabular}

Fonte: Levantamento de processos julgados na $2^{2}$ Vara de Fazenda Pública Privativa do TJDFT - 2005 a 2010.

Nota: Os valores foram arredondados.

critórios modelo e advocacia do Ministério Público Federal ocorrem apenas esporadicamente. Outro indicador de classe social dos demandantes utilizado em estudos nacionais é o tipo de 
serviço de saúde de onde parte o pedido médico dos bens e serviços de saúde demandados, avaliado pela origem da receita médica que acompanhou o pedido inicial ao juiz. Mais uma vez as evidências sugerem que a judicialização não é um fenômeno exclusivo das elites. Apenas 9\% dos processos parte de pedidos obtidos por meio da medicina privada. Não é possível dizer, no entanto, se esse é um caso de validação das receitas de medicina privada por serviços públicos de saúde para o início do litígio judicial.

Embora exista um debate sobre a classe social dos demandantes em diferentes processos de judicialização da saúde no Brasil, uma pergunta sobre classes só faz sentido quando a intenção é contestar os princípios de universalidade que regem o sistema de saúde. Para um sistema que, por ser universal, não discrimina atendimentos por atributos das pessoas, a origem social dos indivíduos deve ser considerada irrelevante. $\mathrm{O}$ que parece ser mais razoável questionar, porém, é se as demandas expressam necessidades de saúde - aquelas que o sistema público de saúde deve atender por imposição constitucional.

Avaliar a necessidade de bens e serviços de saúde a partir de processos judiciais é uma tarefa mais difícil do que pode parecer à primeira vista. No entanto, há informações no curso dos processos que trazem indicações importantes sobre a razoabilidade do que é demandado. Como se verá a seguir, o conjunto formado pelos motivos para o não julgamento em sentença terminativa de processos, as razões para que esse julgamento não tenha ocorrido e o tipo de bens de saúde solicitados indica que a judicialização expressa solicitações para a cobertura de necessidades específicas e urgentes de saúde.

A primeira tabela traz ainda os dados de comprovação de renda disponíveis em 27 processos ( $7 \%$ do total). A renda mais alta comprovada foi de $\mathrm{R} \$ 3.809,00$ e a mais baixa de $\mathrm{R} \$ 354,05$. Vale notar que uma análise da documentação apresentada na entrada do processo indica que $7 \%$ dos requerentes eram analfabetos e $20 \%$ dos processos baseiam seus argumentos na pobreza dos requerentes. Entretanto, considerando que a comprovação não ocorre na maioria dos processos, pode haver uma concentração dos indivíduos com menor renda entre aqueles que apresentaram documento de comprovação no pedido inicial. Por outro lado, a simples exigência ou demanda espontânea de apresentação de comprovação de renda na petição inicial deve ser um dado analisado com cautela: se o Sistema Único de Saúde é universal e não focalizado por classe so- cial, não haveria porque considerar a renda um critério argumentativo para a judicialização de necessidades de saúde.

A Tabela 2 traz os bens de saúde que foram demandados judicialmente. O principal bem judicializado foi acesso à UTI na rede privada de saúde (66\%). Outro bem cuja solicitação judicial foi frequente é a demanda por assistência médica (13\%), porém com menor frequência que o acesso à UTI. A demanda por medicamentos, principal motivo da judicialização na literatura nacional foi provocada por $15 \%$ dos indivíduos. Não há na literatura nacional sobre judicialização da saúde evidências que se comparem ao encontrado no DF, o que sugere ser a judicialização uma resposta às particularidades da política de saúde pública local e suas fragilidades.

As doenças mais comuns são doenças do aparelho circulatório (28\%) e doenças do aparelho respiratório (24\%), conforme Tabela 3. Até 2007 , as demandas por UTI respondiam a $32 \%$ do total de demandas. A partir de 2008, elas passam a representar $71 \%$ do total das demandas.

O padrão de demanda por bens de saúde sugere ser consistente com os diagnósticos médicos apresentados nos pedidos judiciais. Esses diagnósticos apontam para uma prevalência de doenças agudas (78\%) no conjunto dos casos. Os tratamentos de doenças agudas são, em geral, bem enquadrados nos protocolos terapêuticos do sistema público de saúde. Deve ser notado que esses diagnósticos foram realizados quase que integralmente por médicos da rede pública de saúde. A ausência desse tratamento, refletida na judicialização, pode ser um indicador de

Tabela 2. Bens de saúde demandados.

\begin{tabular}{lrr}
\hline \multicolumn{1}{c}{ Demanda } & \multicolumn{2}{c}{ Total } \\
\hline Vaga em UTI na Rede Privada de Saúde & 254 & 66 \\
Medicamentos & 60 & 15 \\
Assistência médica & 52 & 13 \\
Produtos para saúde & 20 & 5 \\
Custos com atendimento na rede privada & 14 & 3 \\
Alimentos & 3 & 1 \\
Tipos específicos de instalação & 3 & 1 \\
Vaga em UTI na Rede Pública de Saúde & 2 & 0 \\
Outros & 3 & 1 \\
\hline
\end{tabular}

Fonte: Levantamento de processos julgados na $2^{2}$ Vara de Fazenda Pública Privativa do TJDFT - 2005 a 2010.

Nota: um processo pode demandar mais de um bem, portanto a soma dos totais pode ultrapassar $100 \%$. 
Tabela 3. Diagnósticos das doenças dos demandantes.

\begin{tabular}{|c|c|c|c|c|c|c|}
\hline \multirow[b]{2}{*}{ Diagnóstico } & \multicolumn{2}{|c|}{ Agudas } & \multicolumn{2}{|c|}{ Crônicas } & \multicolumn{2}{|c|}{ Total } \\
\hline & No & $\%$ & $\mathrm{~N}^{\circ}$ & $\%$ & No & $\%$ \\
\hline Doenças do aparelho circulatório & 54 & 21 & 18 & 8 & 72 & 29 \\
\hline Doenças do aparelho respiratório & 61 & 24 & 1 & 0 & 62 & 24 \\
\hline Diagnósticos inconsistentes & 28 & 11 & 0 & 0 & 28 & 11 \\
\hline Doenças do aparelho digestivo & 12 & 5 & 7 & 3 & 19 & 8 \\
\hline Neoplasias & 1 & 0 & 14 & 6 & 15 & 6 \\
\hline Causas externas (acidentes) & 11 & 4 & 0 & 0 & 11 & 4 \\
\hline Doenças do período neonatal & 8 & 3 & 1 & 0 & 9 & 3 \\
\hline Doenças Infecciosas & 5 & 2 & 3 & 1 & 8 & 3 \\
\hline Doenças renais & 3 & 1 & 2 & 1 & 5 & 2 \\
\hline Doenças neurológicas & 1 & 0 & 4 & 2 & 5 & 2 \\
\hline Doenças endócrinas & 2 & 1 & 2 & 1 & 4 & 2 \\
\hline Doenças hematológicas & 2 & 1 & 1 & 0 & 3 & 1 \\
\hline Malformações congênitas & 2 & 1 & 1 & 0 & 3 & 1 \\
\hline Sintomas não especificados & 5 & 2 & 0 & 0 & 5 & 1 \\
\hline Lesões por causas externas & 2 & 1 & 0 & 0 & 2 & 1 \\
\hline Gravidez e parto & 3 & 1 & 0 & 0 & 3 & 1 \\
\hline Transtornos mentais & 0 & 0 & 1 & 0 & 1 & 0 \\
\hline Total & 200 & 78 & 55 & 22 & 255 & 100 \\
\hline
\end{tabular}

Fonte: Levantamento de processos julgados na 2a Vara de Fazenda Pública Privativa do TJDFT - 2005 a 2010.

reação à omissão das políticas do que demanda por tratamentos não oferecidos pelo sistema.

A Tabela 4 apresenta os argumentos utilizados para fundamentar os processos. Um mesmo processo pode lançar mão de mais de um argumento. Em cada processo a estrutura argumentativa segue um padrão particular sendo, portanto, difícil determinar quais seriam os argumentos centrais e quais os periféricos. No entanto, como conjunto de leis e princípios constitucionais as quais os processos podem recorrer é razoavelmente limitado, foi possível identificar e classificar os argumentos em 5 categorias.

Praticamente todos os processos fundamentam sua argumentação no direito à saúde (95\%) e muitos recorrem ao direito à vida (50\%), ambos difíceis de contestar do ponto de vista jurídico - embora a decisão dos juízes possa variar, é difícil contrapor um argumento baseado no direito à vida com uma argumentação fundamentada na reserva do possível, por exemplo. Relacionado ao fato de que a maioria dos processos solicita vagas em UTI, muito comuns são também os argumentos de risco de vida (76\%), dano irreparável ou de difícil reparação (52\%) e risco de agravo da doença (18\%). E, como já mencionado, o argumento da insuficiência de renda é mencionado em $20 \%$ dos processos.
Tabela 4. Principais argumentos do pedido do requerente.

\begin{tabular}{|c|c|}
\hline Argumento & Total \\
\hline Direito à saúde & $36795 \%$ \\
\hline Risco de vida & $29376 \%$ \\
\hline $\begin{array}{l}\text { Risco de dano irreparável ou de difícil } \\
\text { reparação }\end{array}$ & $20152 \%$ \\
\hline Direito à vida & $19150 \%$ \\
\hline Insuficiência de renda & $7920 \%$ \\
\hline
\end{tabular}

Fonte: Levantamento de processos julgados na $2^{\mathrm{a}}$ Vara de Fazenda Pública Privativa do TJDFT - 2005 a 2010.

Nota: um processo pode demandar mais de um bem, portanto a soma dos totais pode ultrapassar $100 \%$.

Dada a urgência dos tipos de demanda predominantes, a maior parte dos requerentes solicita decisão liminar. Os juízes tendem a conceder essas liminares (70\%). Apenas 8\% das decisões são desfavoráveis aos requerentes, como mostra a Tabela 5. Como predominam demandas por vagas de UTI de indivíduos correndo risco de vida ou agravamento de condição clínica, esse é um resultado de certo modo esperado. 
Tabela 5. Decisão liminar.

\begin{tabular}{lcc}
\hline & N & \% \\
\hline Liminar & 268 & 70 \\
$\quad$ Favorável & 49 & 13 \\
Sem decisão liminar & 31 & 8 \\
Desfavorável ao requerente & 24 & 6 \\
Sem informação & 13 & 3 \\
Favorável parcialmente & $\mathbf{3 8 5}$ & $\mathbf{1 0 0}$ \\
Total & & \\
Fundamentos para liminar desfavorável ao & & \\
requerente & 12 & 38 \\
$\quad$ Ausência de documentos que comprovem & & \\
a urgência & 3 & 11 \\
Lista de espera & 3 & 11 \\
Racionalidade da prescrição médica & 2 & 6 \\
Custo do medicamento/reserva do possível & 2 & 6 \\
Ausência de risco & 2 & 6 \\
Ausência de perícia médica judicial & 7 & 22 \\
Outros & $\mathbf{3 1}$ & $\mathbf{1 0 0}$ \\
Total &
\end{tabular}

Fonte: Levantamento de processos julgados na 2a Vara de Fazenda Pública Privativa do TJDFT - 2005 a 2010.

Nota: Os valores foram arredondados.
A Tabela 5 também decompõe a fração de liminares desfavoráveis aos requerentes segundo o fundamento da decisão liminar. A razão principal para a recusa à concessão de liminares é a ausência de comprovação da urgência do caso. É possível observar na tabela que preocupações com impactos orçamentários das decisões, majoritariamente referentes ao custo de medicamentos, (argumento da reserva do possível) têm pouca importância no total haja vista que em apenas 2 processos $(6 \%)$ o indeferimento da liminar se fundamentou neste argumento.

O estudo trouxe importantes informações a respeito da judicialização da saúde. Como resultados obtidos destaca-se que: (i) do total dos processos levantados $89 \%$ concentram-se nos 3 (três) últimos anos da pesquisa (2007-2010); (ii) o intervalo da faixa etária dos demandantes varia de recém-nascidos a idosos acima de 80 (oitenta) anos; (iii) $85 \%$ dos processos tem origem em receituário da rede pública de saúde e que 95\% dos processos advêm da defensoria pública; (iv) 7\% dos demandantes comprovaram renda na inicial e, desses, $70 \%$ atestaram renda inferior a $\mathrm{R} \$$ 999,00; (v) 66\% das ações tem como pedido a internação em uma vaga em UTI na rede privada de saúde e apenas $15 \%$ dos processos tinha como bem demandado a aquisição de medicamentos; (vi) há uma concentração do diagnóstico em doenças de caráter agudo, representando $78 \%$ dos casos; (vii) $95 \%$ dos processos utiliza o argumento do direito à saúde como fundamentação, seguido dos argumentos de risco de vida e risco de dano irreparável ou de difícil reparação (76\% e 52\%); (viii) em 70\% dos casos a decisão liminar é favorável ao requerente e a negativa ocorre em apenas $8 \%$ dos processos; (ix) 38\% das negativas a decisão judicial baseouse na ausência de documentos que comprovassem a urgência da solicitação e somente em 2 casos $(6 \%)$ foi utilizado o argumento de custo do medicamento e/ou reserva do possível para não conceder a liminar.

\section{Conclusão}

Os resultados obtidos não corroboraram algumas das teses mais difundidas na literatura brasileira como a elitização do processo judicial e a prevalência de requisições de medicamentos de alto custo. A elitização do mecanismo de obtenção de serviços ou produtos através do Poder Judiciário não foi ratificada no estudo de caso analisado. A representação judicial e médica originária de serviços públicos, combinada com a renda comprovada em parte dos processos, é um indício de que a afirmação de elitização não pode ser sustentada em todos os estados brasileiros, já que os dados obtidos no Distrito Federal tendem a confrontar a afirmação.

$\mathrm{O}$ outro argumento apresentado em artigos nacionais quanto à prevalência de utilização da judicialização como meio de adquirir medicamentos de alto custo não inclusos nas políticas públicas de saúde também não foi demonstrado através dos dados. O objeto majoritário de pedidos referia-se a internações em unidades de tratamento intensivo. Fato esse que oferece suporte às decisões judiciais de urgência no deferimento do pedido. Como conclusão avalia-se que são necessários mais estudos com evidências sobre a judicialização da saúde para que seja plausível afirmar teses gerais sobre o tema. A pesquisa realizada traz indícios de que não é possível afirmar que o processo de judicialização da saúde é um movimento da elite econômica brasileira e que o objeto principal das demandas é a aquisição de medicamentos. 


\section{Colaboradores}

D Diniz, TRC Machado e J Penalva participaram igualmente de todas as etapas de elaboração do artigo.

\section{Agradecimentos}

Ao Dr. Alvaro Ciarlini, juiz da 2a Vara de Fazenda Pública Privativa do TJDFT, por possibilitar acesso aos dados. Ao Observatório da Justiça Brasileira pelo financiamento concedido. Às pesquisadoras Renata Janaina de Sousa Brito, Natália Peres Kornijezuk e Vanessa Carrião pela coleta dos dados. À equipe da ANIS - Instituto de Bioética, Direitos Humanos e Gênero, em especial ao João Neves, pelo tratamento dos dados, e Alberto Madeiro, pela classificação médica dos diagnósticos.

\section{Referências}

1. Barroso LR. Da falta de efetividade à judicialização excessiva: direito à saúde, fornecimento gratuito de medicamentos e parâmetros para a atuação judicial. Revista Interesse Público 2008.

2. Vieira FS, Zucchi P. Distorções causadas pelas ações judiciais à política de medicamentos no Brasil. Rev Saude Publica 2007; 41(2):214-222.

3. Messeder AM, Osório-de-Castro CGS, Luiza VL. Mandados judiciais como ferramenta para garantia do acesso a medicamentos no setor público: a experiência do Estado do Rio de Janeiro, Brasil. Cad Saude Publica 2005; 21(2):525-534.

4. Diniz D, Medeiros M, Schwartz IVD. Consequências da judicialização das políticas de saúde: custos de medicamentos para as mucopolissacaridoses. Cad Saude Publica 2012; 28(3):479-489.

5. Medeiros M, Diniz D. A tese da judicialização da saúde pelas elites: os medicamentos para mucopolissacaridose. Cien Saude Colet 2013; 18(4):1079-1088.

6. Sarmento D. A proteção judicial dos direitos sociais: alguns parâmetros ético-jurídicos. In: Souza Neto CP, Sarmento D. Direitos Sociais: fundamentos, judicialização e direitos sociais em espécie. Rio de Janeiro: Lumen Juris; 2008.

7. Baptista, Tatiana Wargas de Faria; Machado, Cristiani Vieira; e Lima, Luciana Dias de. Responsabilidade do Estado e direito à saúde no Brasil: um balanço da atuação dos Poderes. Cien Saude Colet 2009; 14(3):829-839.

8. Borges DCL. Uma Análise das Ações Judiciais para o Fornecimento de Medicamentos no Âmbito do SUS: o caso do estado do Rio de Janeiro no ano de 2005 [dissertação]. Rio de Janeiro: Fiocruz; 2007.

9. Cosendey MAE, Bermudez JAZ, Reis ALA, Silva HF, Oliveira MA, Luiza VL. Assistência Farmacêutica na atenção básica de saúde: a experiência de três estados brasileiros. Cad Saude Publica 2000; 16(1):171-182.

10. Vieira FS, Zucchi P. Distorções causadas pelas ações judiciais à política de medicamentos no Brasil. Rev Saude Publica 2007; 41(2):214-222.

11. Marques SB, Dallari SG. Garantia do direito social à assistência farmacêutica no Estado de São Paulo. Rev Saude Publica 2007; 41(1):101-107.

12. Kornis G, Braga MH, Zaire C. Os Marcos Legais das Políticas de Medicamentos no Brasil Contemporâneo (1990-2006). Revista APS 2008; 11(1):85-99.

13. Brasil. Tribunal de Contas da União (TCU). Avaliação do TCU sobre a Ação Assistência Financeira para Aquisição e Distribuição de Medicamentos Excepcionais. Brasília: TCU; 2005.

14. Pereira JR, Santos RI, Nascimento Junior JM, Schenkel EP. Análise das demandas judiciais para o fornecimento de medicamentos pela Secretaria de Estado da Saúde de Santa Catarina nos anos de 2003 e 2004. Cien Saude Colet 2010, 15(Supl. 3):3551-3560.

15. Diniz D. Judicialização de medicamentos no SUS: memorial ao STF. Brasília: SérieAnis; 2009. [documento na Internet]. [acessado 2010 maio 19]. Disponível em: http://www.anis.org.br/serie/artigos/ sa66_diniz_medicamentos_stf.pdf 
16. Diniz D. Rationing the access to high cost medicines in developing world countries. Developing World Bioethics 2009; 9(2):2-3.

17. Ham C, Robert G, editors. Reasonable Rationing: International Experience of Priority Setting in Health Care. Maidenhead: Open University Press; 2003.

8. Syrett K. Law, Legitimacy and Rationing of Health Care: a contextual and comparative perspective. Cambridge: Cambridge University Press; 2007.

19. Daniels N, Sabin J. Can We Learn to Share Medical Resources? Setting Limits Fairly. Oxford: Oxford University Press; 2002.

Artigo apresentado em 30/11/2012

Aprovado em 27/02/2013

Versão final apresentada em 07/03/2013 